\title{
Picosecond Fiber Optical Parametric Oscillator with 500 nm Tuning Range Using Dispersion-Shifted Fiber
}

\author{
Yue Zhou, Kim K. Y. Cheung, Sigang Yang, P. C. Chui, and Kenneth K. Y. Wong* \\ Photonic Systems Research Laboratory, Department of Electrical and Electronic Engineering, \\ The University of Hong Kong, Pokfulam, Hong Kong. \\ *E-mail:kywong@eee.hku.hk
}

\begin{abstract}
We demonstrate a picosecond fiber optical parametric oscillator with a tuning range of $500 \mathrm{~nm}$ pumped by a relatively low-cost intensity-modulated pump. Pulses with pulsewidth from $7 \mathrm{ps}$ to $17 \mathrm{ps}$ are generated. (C)2010 Optical Society of America

OCIS codes: (060.4370) Nonlinear Optics, fibers; (190.4380) Nonlinear optics, four-wave mixing
\end{abstract}

\section{Introduction}

Picosecond optical pulse sources have been playing an important role in ultrafast processes and optical communications [1]. However, high-quality picosecond laser is generally not available at some non-conventional wavelengths due to the lack of efficient gain medium. Thanks to the ultra-fast response, wide-gain bandwidth [2], high gain [3] and large detune from the pump [2] of fiber optical parametric amplifier (FOPA), efficient short pulse generation is possible at non-conventional wavelengths.

Previous effort included utilizing conventional parametric gain to generate picosecond pulse over C/L-band [4]. In our previous work, we also demonstrated the generation of picosecond pulse at large wavelength detune $(\sim 100$ $\mathrm{nm}$ ) from the pump using a continuous-wave (CW) signal as a seed [5]. However, the availability of CW lasers at such wavelength $(\sim 1640 \mathrm{~nm})$ or even longer wavelength limits its application. Therefore it is highly desirable to explore its corresponding fiber optical parametric oscillator (FOPO) configuration, which does not require any seeding light [6-10]. In our previous work [11], picosecond FOPO with a $250 \mathrm{~nm}$ tuning range around $1550 \mathrm{~nm}$ was demonstrated, which was synchronously pumped by a mode-locked fiber laser (MLFL). However, a MLFL is relatively expensive, so it is desirable to build a picosecond source simply from a CW pump.

In this paper, we demonstrate a fully fiber-integrated picosecond FOPO based on dispersion-shifted fiber (DSF). A tuning range of $500 \mathrm{~nm}$ is achieved by changing the pump wavelength from $1532 \mathrm{~nm}$ to $1549 \mathrm{~nm}$. Pulses with pulsewidth from 7 ps to $17 \mathrm{ps}$ are generated. This scheme has the potential to be a cost-effective source in generating short pulse for ultra-fast optical communications outside the conventional wavelength window.

\section{Experimental Setup}

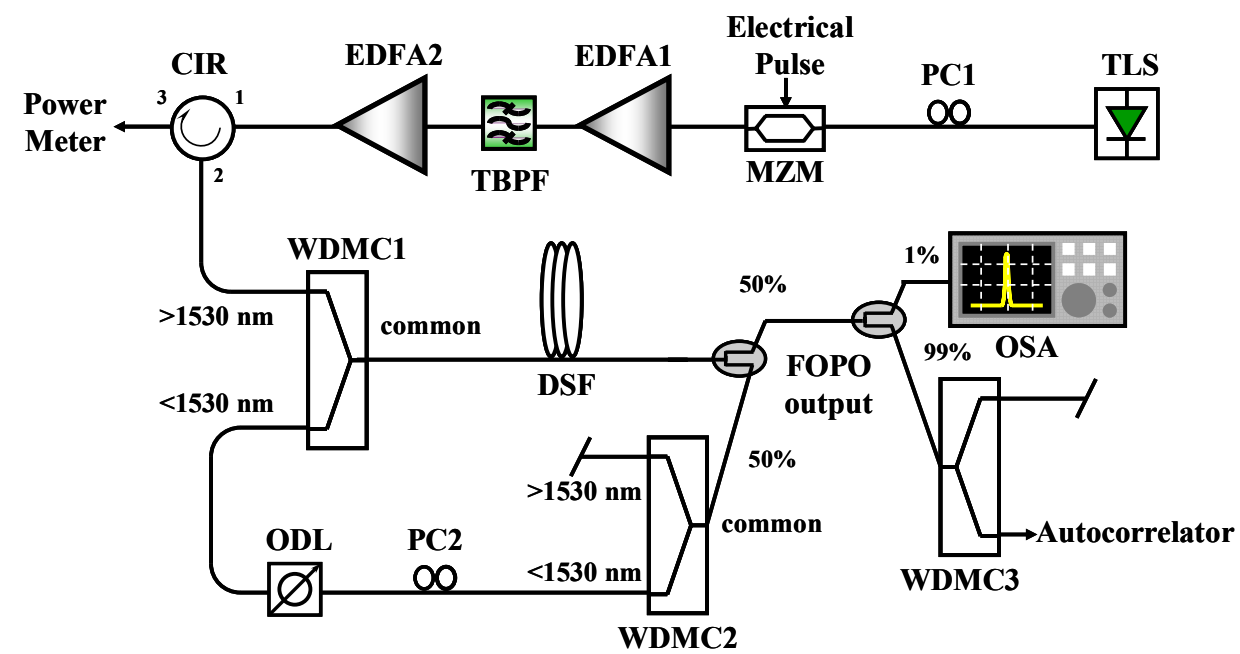

Fig. 1. Experimental setup of the FOPO. EDFA: erbium-doped fiber amplifier, TBPF: tunable band-pass filter, OSA: optical spectrum analyzer, MZM: Mach-Zehnder modulator, ODL: optical delay line, CIR: circulator. 
The experimental setup of widely-tunable picosecond pulse generator is shown in Fig.1. The pump source is a tunable laser source (TLS), which is tuned from $1532 \mathrm{~nm}$ to $1549 \mathrm{~nm}$ to achieve the widest tuning range. The CW output of the TLS is intensity-modulated by a $10 \mathrm{MHz}$ electrical pulse with duty ratio of $1 / 1000$ to produce optical pump pulse with pulsewidth of $100 \mathrm{ps}$ and repetition rate of $10 \mathrm{MHz}$. Polarization controller PC1 aligns the pump's state of polarization (SOP) with the transmission axis of the MZM. The pump is then amplified by EDFA1, filtered by a 1-nm bandwidth TBPF and further amplified by EDFA2 to produce a high-power, low-noise pump. It then passes through a circulator and the reflected power is observed using a power meter. As measured from the power meter, the stimulated Brillouin scattering (SBS) is low and therefore the phase modulator used in [8] is not required to suppress the SBS. It is then coupled into the cavity for parametric amplification through a wavelength-division multiplexing coupler (WDMC1), which has a cutoff wavelength of $1530 \mathrm{~nm}$. The peak power of the pump is measured to be $25 \mathrm{~W}$ at the fiber input. The gain medium inside the cavity is a spool of 40-m dispersion-shifted fiber (DSF), which has nonlinear coefficient of $2 \mathrm{~W}^{-1} \mathrm{~km}^{-1}$, zero-dispersion wavelength (ZDW) of $1548.7 \mathrm{~nm}$, and dispersion slope of $0.0693 \mathrm{ps} / \mathrm{nm}^{2} / \mathrm{km}$. A $50 / 50$ coupler in the cavity provided $50 \%$ feedback and $50 \%$ output. The feedback branch is filtered by the WDMC2 with a cutoff wavelength of $1530 \mathrm{~nm}$, so that only the signal (shorter wavelength component) returns to the DSF through WDMC1, and the idler (longer wavelength component) is blocked. As a result, the FOPO is only singly resonant with the signal. The PC2 in the cavity is used to align the SOP of the signal with that of the pump. The total length of the discrete standard single mode fiber (SMF) used to connect the components in the cavity is $20 \mathrm{~m}$, so the total cavity length is $60 \mathrm{~m}$.

There are two conditions need to be satisfied for stable oscillations. One is that the signal pulse should synchronize with the pump pulse, so we need to use an ODL inside the cavity to adjust the cavity length thus the round trip time of the signal matches the pump repetition rate. Since the round trip time of different signal is wavelength dependent because of group-velocity dispersion (GVD), each tuning we need to tune the ODL to synchronize the signal at oscillating wavelength with the pump. The other condition is that the single-pass signal gain should be larger than the cavity loss. The round trip loss of the cavity is measured to be $9.5 \mathrm{~dB}$.

The single-pass signal peak gain of the FOPO in the case of perfect phase matching can be calculated using [7]:

$$
G_{d B} \approx 10 \log _{10}\left[\frac{1}{4} \exp (2 \gamma P L)\right]
$$

where $L$ is the fiber length, $P$ is the peak power of the pump, $\gamma$ is the nonlinear coefficient. The peak power corresponding to a $9.5-\mathrm{dB}$ gain is calculated to be $22.3 \mathrm{~W}$. The experimental threshold pump peak power is $25 \mathrm{~W}$, matches reasonably well with the calculated value. After the above two conditions are satisfied, stable oscillations can be obtained. The FOPO output spectrum is monitored by an OSA through a 1/99 coupler. WDMC3 is used to filter out the desired signal or idler. The pulsewidth of the signal (idler) is measured using an autocorrelator.

\section{Results and Discussion}

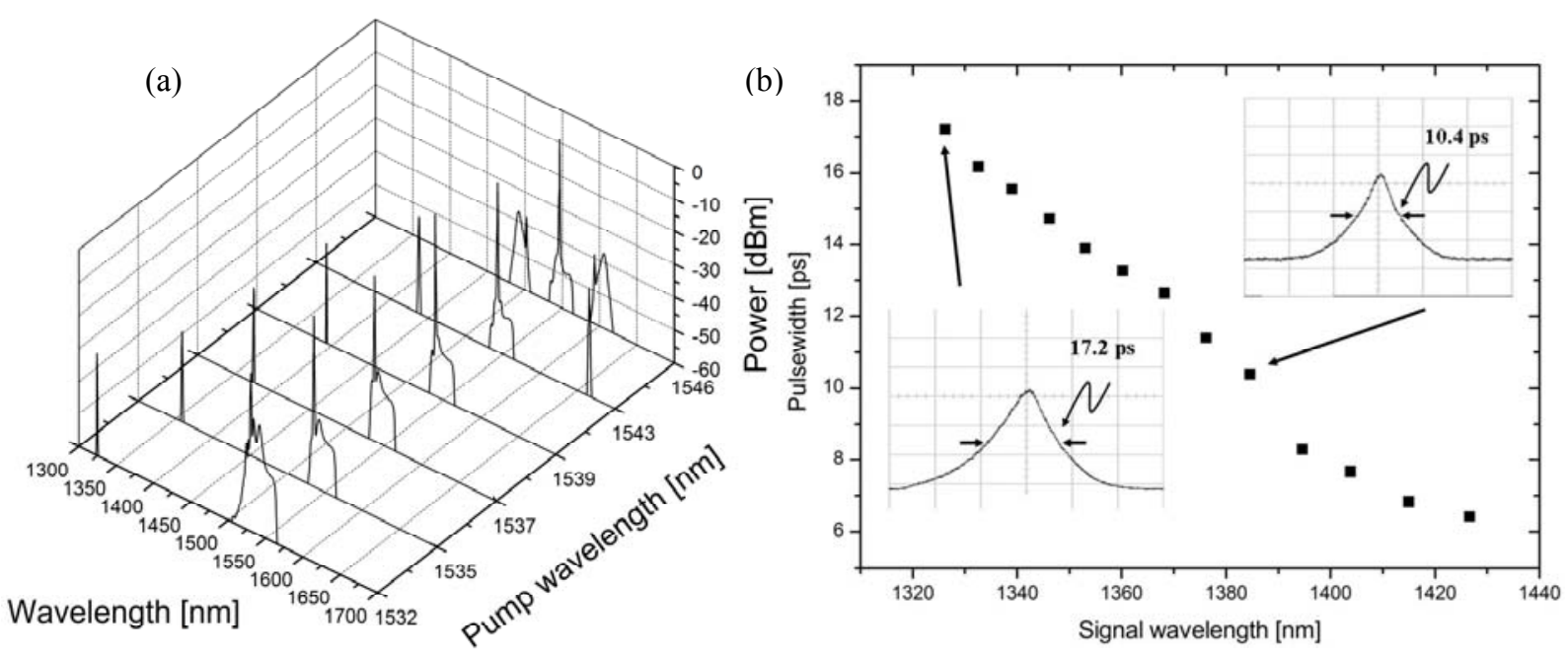

Fig. 2. (a) Optical spectra measured at FOPO output using OSA, (b) Signal pulsewidth versus wavelength. Insets are autocorrelation traces at some wavelengths.

Fig. 2 (a) shows the optical spectra measured at the FOPO output port. Tunability is achieved by tuning the pump wavelength from $1549 \mathrm{~nm}$ to $1532 \mathrm{~nm}$, and the pump power is slightly adjusted to maintain almost the same for all 
spectrum peaks. As the pump wavelength is decreased, the detuning of the sidebands increases. When the ODL is tuned at the same time to synchronize the signal with the pump, the achievable output tuning range is from $1320 \mathrm{~nm}$ to $1700 \mathrm{~nm}$ measured by the OSA. Since the operating wavelength of our OSA ends at $1700 \mathrm{~nm}$, the idler beyond this wavelength is not shown in Fig. 2 (a). The longest idler wavelength calculated from the experimental measured shortest signal wavelength is $1820 \mathrm{~nm}$. Therefore, the tuning range of our FOPO is from $1320 \mathrm{~nm}$ to $1820 \mathrm{~nm}$, which is as wide as $500 \mathrm{~nm}$. The tuning range is larger than those picosecond FOPOs reported in [9, 11]. There are three factors which may combine to limit the further enhancement of the tuning range. Firstly, the DSF is not polarization maintained, and random birefringence may decrease the four-wave mixing (FWM) efficiency at large detuning [12]; secondly, the fluctuations of the ZDW along the fiber will also limit the tuning range of this kind of FOPO [13]; the third factor is the walk-off between the signal and pump, which increases with their wavelength separation and thereby decreases their interaction length.

Fig. 2 (b) shows the output pulsewidth as a function of signal wavelength. Insets are autocorrelation traces at some wavelengths measured by the autocorrelator. The real full-width half-maximum (FWHM) pulsewidth is calculated by assuming a sech ${ }^{2}$ pulse shape, multiplied the FWHM correlation width using a deconvolution factor of 0.648. The pulsewidth increases from $7 \mathrm{ps}$ to $17 \mathrm{ps}$ when the signal detunes further from the pump, thus the walk-off between the signal and the pump becomes larger which broaden the signal pulsewidth. The output pulses are narrower than the pump pulses (100 ps) because of the pulse narrowing effect of FOPA [4].

\section{Conclusion}

In conclusion, an all-fiber widely-tunable picosecond optical parametric oscillator based on DSF was demonstrated. The output was continuously tunable from $1320 \mathrm{~nm}$ to $1820 \mathrm{~nm}$, when the pump wavelength was tuned between $1549 \mathrm{~nm}$ to $1532 \mathrm{~nm}$. Pulses were generated with pulsewidth from 7 ps to $17 \mathrm{ps}$. This scheme has the potential to be a cost-effective source in generation short pulse for ultrafast communication in the non-conventional wavelength bands.

\section{Acknowledgment}

The work described in this paper is partially supported by grants from the Research Grants Council of the Hong Kong Special Administrative Region, China (Project No. HKU 7179/08E and HKU 7183/09E).

\section{References}

[1] P.T. Ho, L. A. Glasser, E. P. Ippen, and H. A. Haus, "Picosecond pulse generation with a cw GaAlAs laser diode," Appl. Phys. Lett. 33, 241242 (1978).

[2] M. E. Marhic, K. K.-Y. Wong and L. G. Kazovsky, "Wide-band tuning of the gain spectra of one-pump fiber optical parametric amplifiers," IEEE J. Select. Topics. Quant. Electron. 10, 1133-1141 (2004).

[3] T. Torounidis, P.A. Andrekson, B.-E. Olsson, "Fiber-optical parametric amplifier with 70-dB gain," IEEE Photon. Technol. Lett. 18, 1194 1196 (2006).

[4] T. Torounidis, M. Karlsson, and P. A. Andrekson, "Fiber Optical Parametric Amplifier Pulse Source: Theory and Experiments," J. Lightwave Technol. 23, $4067-4073$ (2005).

[5] B. P. P. Kuo, K. K. Y. Cheung, Y. Zhou and K. K. Y. Wong, "Wide-band generation of picosecond pulse via idler generation in optical parametric amplifier," in 2009 IEEE/LEOS Winter Topical Meetings, Innsbruck, Austria, paper TuC3.5, 2009.

[6] J. E. Sharping, "Microstructure Fiber Based optical parametric oscillators," J. Lightwave Technol. 26, $2184-2191$ (2008).

[7] G. K. L. Wong, S. G. Murdoch, R. Leonhardt, J. D. Harvey, and V.Marie, "High-conversion-efficiency widely-tunable all-fiber optical parametric oscillator," Opt. Express, 15, 2947-2952 (2007).

[8] Y. Q. Xu, S. G. Murdoch, R. Leonhardt, J. D. Harvey, "Raman-assisted continuous-wave tunable all-fiber optical parametric oscillator," J. Opt. Soc. Am. B, 26, 1351-1355 (2009).

[9] Y. Deng, Q. Lin, F. Lu, G. Agrawal, and W. Knox, "Broadly tunable femtosecond parametric oscillator using a photonic crystal fiber," Opt. Lett, 30, 1234-1236 (2005).

[10] J. E. Sharping, M. Fiorentino, P. Kumar, and R. S. Windeler, "Optical-parametric oscillator based on four-wave mixing in microstructure fiber," Opt. Lett. 27, 1675-1677 (2002).

[11] Y. Zhou, K. K. Y. Cheung, S. Yang, P. C. Chui and K. K. Y. Wong, "Widely-tunable picosecond optical parametric oscillator using highlynonlinear fiber", Opt. Lett., 34, 989-991, 2009.

[12] C. McKinstrie, H. Kogelnik, R. Jopson, S. Radic, and A. Kanaev, "Four-wave mixing in fibers with random birefringence," Opt. Express, 12, 2033-2055, 2004.

[13] J. S. Y. Chen, S. G. Murdoch, R. Leonhardt, and J. D. Harvey, "Effect of dispersion fluctuations on widely tunable optical parametric amplification in photonic crystal fibers," Opt. Express, 14, 9491-9501, 2006. 